\title{
Approach to Frontal Sinus Outflow Tract Injury
}

\author{
Yong Hyun Kim, \\ Baek-Kyu Kim \\ Department of Plastic and Reconstructive \\ Surgery, Seoul National University Bundang \\ Hospital, Seoul National University College of \\ Medicine, Seongnam, Korea
}

No potential conflict of interest relevant to this article was reported.
Frontal sinus outflow tract (FSOT) injury may occur in cases of frontal sinus fractures and nasoethmoid orbital fractures. Since the FSOT is lined with mucosa that is responsible for the path from the frontal sinus to the nasal cavity, an untreated injury may lead to complications such as mucocele formation or chronic frontal sinusitis. Therefore, evaluation of FSOT is of clinical significance, with FSOT being diagnosed mostly by computed tomography or intraoperative dye. Several options are available to surgeons when treating FSOT injury, and they need to be familiar with these options to take the proper treatment measures in order to follow the treatment principle for FSOT, which is a safe sinus, and to reduce complications. This paper aimed to examine the surrounding anatomy, diagnosis, and treatment of FSOT.

Keywords: Frontal sinus / Frontonasal / Recess / Duct

\section{INTRODUCTION}

Frontal sinus fracture and nasoethmoid orbital fracutre accounts for approximately $10 \%$ of all craniofacial fractures and it occurs from high velocity impact with the main cause of injury being motor vehicle crashes or assault. Approximately $1 / 3$ or more cases $(25 \%-50 \%)$ of frontal sinus fractures are accompanied by frontal sinus outflow tract (FSOT) injury [1-5].

FSOT is the nasal part of the frontal sinus, and is referred to as the nasofrontal duct or the frontal recess, while the use of various terms and concepts of FSOT has interfered with understanding the anatomy of FSOT. This has also caused confusion in diagnosis and treatment of FSOT injury [6]. Echo et al. [7] stated that since the nasofrontal duct is rarely a distinct duct, nasal frontal outflow tract is the more appropriate term. In this paper, nasofrontal duct and frontal recess will be collectively referred to as FSOT.

To manage frontal sinus fractures successfully, it is important to know the structural pathology of the frontal sinus, including

Correspondence: Baek-Kyu Kim

Department of Plastic and Reconstructive Surgery, Seoul National University Bundang Hospital, Seoul National University College of Medicine, 82 Gumi-ro 173 Beon-gil, Bundang-gu, Seongnam 13620, Korea

E-mail: plasrecon@gmail.com

Received July 25, 2016 / Revised March 4, 2017 / March 4, 2017 the anatomy of the frontal sinus, and it is important to maintain sinus function, restore facial aesthetics, and prevent complications [4]. Being FSOT injury present is of special clinical significance, because complications associated with FSOT injury can include mucocele formation or chronic frontal sinusitis (infection) $[1,3]$. Generally, however, no pathognomonic sign indicates the presence of FSOT injury [1]. Therefore, specific radiographic computed tomography (CT) criteria and intraoperative dye irrigation tests can be used to diagnose the presence of FSOT injuries [3]. Treatment options for FSOT injury include observation, FSOT reconstruction, obliteration, and endoscopic frontal sinus surgery $[3,4,7,8]$.

\section{ANATOMY}

For a successful FSOT surgery, it is important to have a full understanding of the FSOT and its surrounding anatomy (Figs. 1, 2). The FSOT is a complex space that includes the frontal infundibulum where the frontal sinus narrows toward the frontal ostium, the frontal sinus ostium itself, and the frontal recess below the ostium [3]. From a sagittal section view, the FSOT forms an hourglass shaped structure with the frontal infundibulum and frontal ostium. The drainage of the frontal sinus starts in the ostia, which 


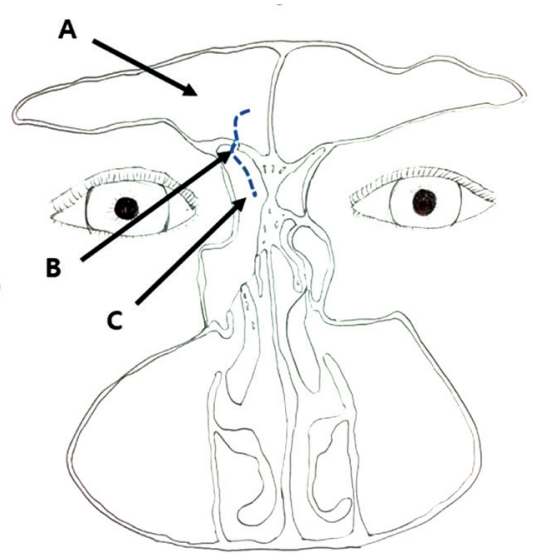

Fig. 1. Coronal CT of FSOT. The dotted line depicts the frontal sinus out flow tract. A, frontal sinus; B, frontal sinus ostium; C, frontal recess; CT, computed tomography; FSOT, frontal sinus outflow tract.

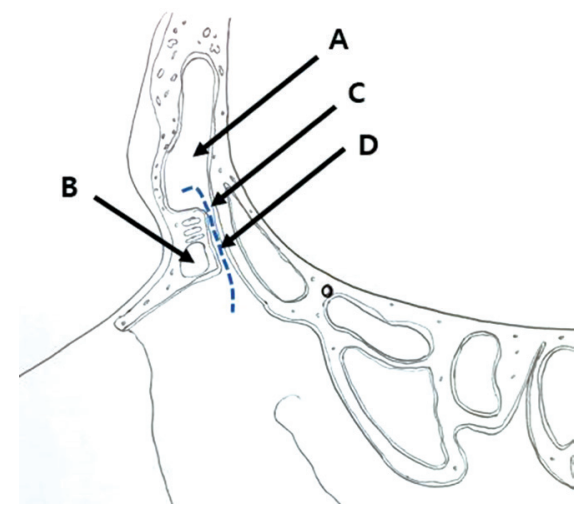

Fig. 2. Sagittal CT of FSOT. The dotted line depicts the frontal sinus out flow tract. A, infundibulum; B, agger nasi cell; $\mathrm{C}$, frontal sinus ostium; D, frontal recess; CT, computed tomography; FSOT, frontal sinus outflow tract.

is bilaterally located in the medial and posterior floor of the frontal sinus, and drainage continues caudally to the nasal cavity through the FSOT [5].

The FSOT is located in the junction of the frontal sinus and in the most anterosuperior part of the ethmoid sinus. Its boundaries are as follows [6,9-11]: (1) Anterior: posterior wall of the agger nasi cell (when present) or the superior portion of the uncinated process. (2) Lateral: lamina papyracea and the uncinated process. (3) Medial: Most anterior/superior portion of the middle turbinate. (4) Posterior: anterior/superior portion of the ethmoidal bulla.

\section{DIAGNOSIS}

FSOT can be diagnosed via plain radiographs, computed tomography, and intraoperative irrigating dye.

\section{Plain radiographs (X-ray)}

For plain skull radiographs, Caldwell and lateral views can be used, but plain radiographs alone are limited in evaluating FSOT, which is because it only evaluates limited parts of the posterior table of the frontal sinus [1]. FSOT injury can be indirectly surmised by the position of the frontal sinus fracture line, but doing so is limited. The presence of a frontal sinus fracture can be suspected based on air-fluid levels of the frontal sinus, a diffusely cloudy sinus, and pneumocephalus [5].

\section{Computed tomography}

CT is the gold standard for evaluating frontal sinus injuries. Axial images on CT are useful for evaluating the anterior and posterior tables of the frontal sinus, while coronal images on CT are useful for evaluating the frontal sinus floor and orbital roof. Although the patency of FSOT can be evaluated from sagittal images, it is unfortunate that evaluation of the involvement of FSOT is not easy $[4,8]$.

FSOT injury can be suspected when the following findings are seen on CT: when there is a fracture (1) in the medial portion of the frontal sinus, (2) in the anterior ethmoidal complex, and (3) of the floor of the frontal sinus [5]. This is because the FSOT is located in the medial part of the frontal sinus and runs to the anterior ethmoid complex, while the ostia of the FSOT is located in the base of the frontal sinus [1,7].

CT can be used not only to diagnose FSOT injury, but also to establish treatment plans and for follow-up reviews of patients with frontal sinus injury. CT can also be used to check for spontaneous sinus ventilation to diagnose complications such as mucocele [4]. 


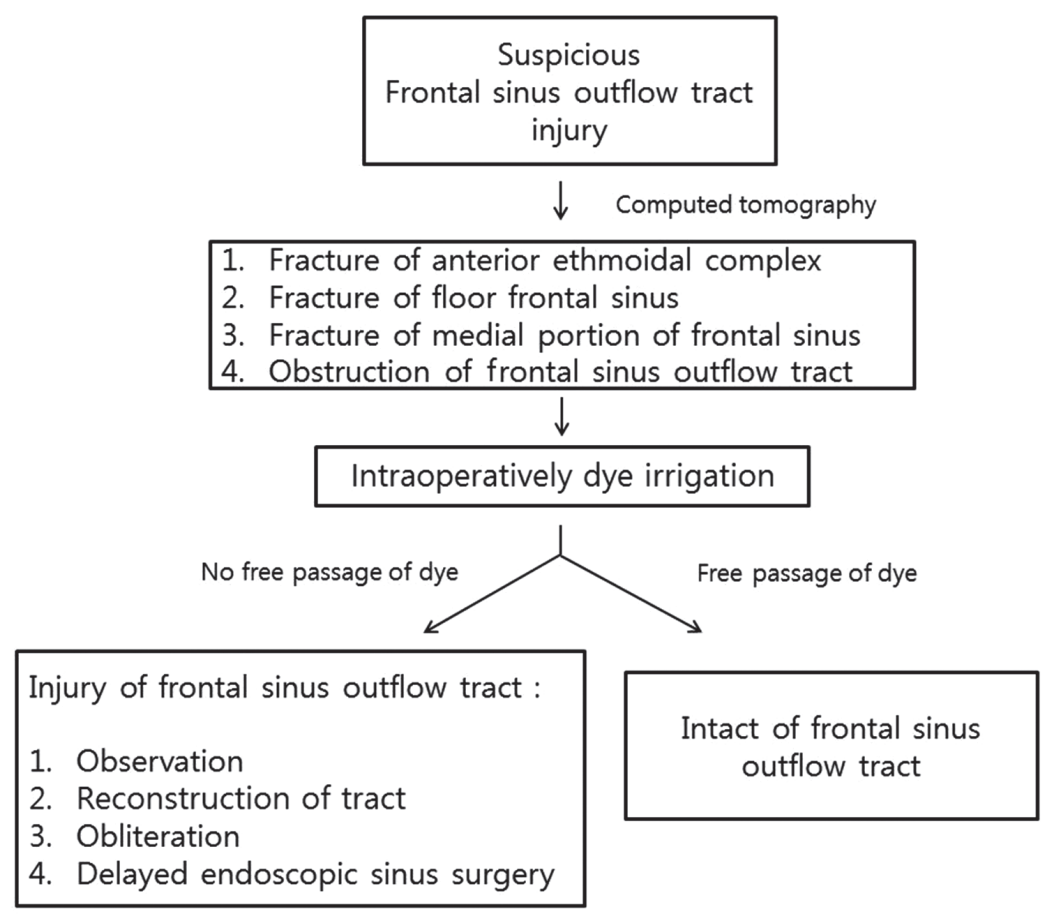

Fig. 3. Treatment algorithm of frontal sinus outflow tract injury.

\section{Intraoperatively assessment (diluted methy- lene blue, fluorescein)}

Intraoperatively, confirmative diagnosis can be made via irrigating dye marker solution and FSOT injury can be confirmed if disruption of free passage into the nasal cavity is found by irrigation into the frontal sinus with $5 \mathrm{~mL}$ of a 1:1 dilution of methylene blue and normal saline or fluorescein [1]. The passage of dye can be confirmed by putting sponge or cotton into the middle meatus level to check for staining and if the dye is not noted, it can be determined that the FSOT is disrupted or occluded. In addition, delayed fibrosis or new bone formation with osteitis may result in narrowing or obstruction of FSOT, even if passage of dye is performed at the early stage of trauma, even if bone fragments or lacerated mucosa remains [5].

\section{TREATMENT}

The treatment principle of FSOT injury is a safe sinus and reducing the long-term risk of complications [4]. In cases with frontal sinus fracture without FSOT injury, open reduction and internal fixation are performed depending on whether there is a bony displacement $[4,5,7]$.

Three treatment options can be considered for frontal sinus fracture with definitive FSOT injury. The first option is surgical enlargement of the frontal ostium for maintaining the patency of the FSOT, which involves insertion of stents or reconstruction using a mucoperiosteal flap. The second option is preventing mucocele formation and infection through frontal sinus obliteration [3]. The third option is to make a clinical evaluation of the presence of frontal sinus ventilation while observing over time via serial CT [2]. Medical treatments are administered during the observation period, and after 4 weeks of observation, if obstruction of FSOT is ultimately confirmed, then the FSOT is recanalized by endoscopic frontal sinus surgery (Fig. 3) [3,4].

\section{FSOT reconstruction}

If there is an injury to the FSOT, drainage from the frontal sinus to the nasal cavity is obstructed, which can be treated by inserting 
a stent to reconstruct the obstructed FSOT. Reconstruction can be performed using stent materials that include alloplastic materials such as rubber, gold, or silicone, or with autoplastic materials such as a mucoperiosteal flap. The inserted stent is left for several weeks and up to $30 \%$ of the patients experience failure in maintaining the patency of the FSOT after removing the stent, which is caused by restenosis from postoperative scarring [5].

\section{Obliteration}

Obliteration involves using a surgical curettage to remove the mucosa of the internal wall in the frontal sinus, followed by blockage of the FSOT by an autogenous bone graft, temporalis muscle plugs, or pericranial or galeal flaps. For successful frontal sinus obliteration, communication between the frontal sinus and nasal cavity is blocked to prevent the formation of mucocele or mucopyocele, minimize dead space in the frontal sinus, and reduce the risk of infection by preventing accumulation of blood and fluid [5].

While obliteration is widely used during frontal sinus injury, it also has several disadvantages such as facial scarring and frontal neuralgia due to surgical injury and donor site morbidity.

\section{Observation and endoscopic sinus surgery}

Although obliteration has been regarded as the gold standard for FSOT injury, observation and delayed endoscopic sinus surgery are gaining attention because of recent advancements in endoscopic surgery and enhanced CT imaging. Even with an injury to the FSOT, observation is possible when clinical examination and serial CT imaging show patency of the FSOT. Because complications such as frontal sinusitis do not occur among patients with spontaneous ventilation of the frontal sinus, follow up by CT scans is used to check for mucocele formation or sinus obstruction [3].

During the observation period, patients are administered 4 weeks of topical nasal steroid spray, tapered prednisone therapy, and antibiotics [4].
After 4 weeks of observation, follow up CT can be used to confirm ventilation of the frontal sinus and restoration of mucociliary clearance. If FSOT is obstructed, delayed recanalization of the FSOT can be achieved by endoscopic frontal sinus surgery, such as endoscopic frontal sinusotomy [3,4].

Recent advances in endoscopic surgery have led to the initial debridement of fractured bony segments and lacerated mucosa at the early stage of trauma as well as the delayed procedure [12].

\section{REFERENCES}

1. Heller EM, Jacobs JB, Holliday RA. Evaluation of the frontonasal duct in frontal sinus fractures. Head Neck 1989;11:46-50.

2. Yakirevitch A, Bedrin L, Alon EE, Yoffe T, Wolf M, Yahalom R. Relation between preoperative computed tomographic criteria of injury to the nasofrontal outflow tract and operative findings in fractures of the frontal sinus. Br J Oral Maxillofac Surg 2013;51:799-802.

3. Smith TL, Han JK, Loehrl TA, Rhee JS. Endoscopic management of the frontal recess in frontal sinus fractures: a shift in the paradigm? Laryngoscope 2002;112:784-90.

4. Koento T. Current advances in sinus preservation for the management of frontal sinus fractures. Curr Opin Otolaryngol Head Neck Surg 2012;20:274-9.

5. Tiwari P, Higuera S, Thornton J, Hollier LH. The management of frontal sinus fractures. J Oral Maxillofac Surg 2005;63:1354-60.

6. Kim KS, Kim HU, Chung IH, Lee JG, Park IY, Yoon JH. Surgical anatomy of the nasofrontal duct: anatomical and computed tomographic analysis. Laryngoscope 2001;111:603-8.

7. Echo A, Troy JS, Hollier LH Jr. Frontal sinus fractures. Semin Plast Surg 2010;24:375-82.

8. Strong EB. Frontal sinus fractures. Oper Tech Otolayngol Head Neck Surg 2008;19:151-60.

9. Wormald PJ. The agger nasi cell: the key to understanding the anatomy of the frontal recess. Otolaryngol Head Neck Surg 2003;129:497507.

10. Lien CF, Weng HH, Chang YC, Lin YC, Wang WH. Computed tomographic analysis of frontal recess anatomy and its effect on the development of frontal sinusitis. Laryngoscope 2010;120:2521-7.

11. Zhang L, Han D, Ge W, Xian J, Zhou B, Fan E, et al. Anatomical and computed tomographic analysis of the interaction between the uncinate process and the agger nasi cell. Acta Otolaryngol 2006;126:84552.

12. Emara TA, Elnashar IS, Omara TA, Basha WM, Anany AM. Frontal sinus fractures with suspected outflow tract obstruction: a new approach for sinus preservation. J Craniomaxillofac Surg 2015;43:1-6. 